\title{
Densification of functional plasma polymers by momentum transfer during film growth
}

\author{
Dirk Hegemann, ${ }^{\text {a) }}$ Enrico Körner, ${ }^{\text {b) }}$ Noémi Blanchard, Martin Drabik, \\ and Sébastien Guimond ${ }^{\mathrm{c})}$ \\ Empa, Swiss Federal Laboratories for Materials Science and Technology, \\ Lerchenfeldstrasse 5, 9014 St. Gallen, Switzerland
}

(Received 14 October 2012; accepted 4 November 2012; published online 21 November 2012)

\begin{abstract}
Functional plasma polymers were deposited from pure ethylene discharges and with the addition of carbon dioxide or ammonia. The incorporation of oxygen and nitrogen-containing functional groups depends on the fragmentation in the gas phase as well as on the densification during film growth. While a minimum energy per deposited carbon atom is required for cross-linking, the densification and accompanying reduction of functional group incorporation was found to scale linearly with momentum transfer through ion bombardment during film growth. (C) 2012 American Institute of Physics. [http://dx.doi.org/10.1063/1.4767999]
\end{abstract}

Plasma polymerization is generally governed by (i) fragmentation of a monomer and generation of film-forming species, (ii) transport of film-forming and reactive species to the substrate, (iii) formation of nucleation sites at the substrate surface to support sticking of film-forming species, and (iv) cross-linking, densification, and etching processes during film growth. By control of the gas phase processes (energy input, gas flow) and the surface processes (ion bombardment), the properties of the deposited plasma polymer films can be adjusted over a broad range with respect to, e.g., functional group density, permeability, permanence, and film density. Film density in turn scales with the mechanical properties such as hardness and elastic modulus. ${ }^{1-3}$

The activation and dissociation of the monomer (as well as reactive gases) by electron impact within the active plasma zone depends on the energy invested per particle, which is proportional to the external parameters power input per (monomer) gas flow rate $W / F_{m}{ }^{4}$ Assuming an activation barrier (requiring an activation energy $E_{a}$ ) for a specific plasma chemical reaction pathway to produce film-forming species, a quasi-Arrhenius description can be considered for the mass deposition rate $R_{m}$ within the corresponding regime

$$
\frac{R_{m}}{F_{m}}=C \exp \left(-\frac{E_{a}}{W / F_{m}}\right),
$$

where the pre-factor $C$ depends on the surface loss probability (likelihood that the film-forming species is incorporated into the film growth), the efficiency of power coupling (thus also on the electron energy distribution function), the reaction cross section of the specific reaction pathway, the deposition area, and the molecular mass of the film-forming species. ${ }^{5}$ The pre-factor might be constant over a broad parameter range of $W / F_{m}$, thus enabling the observation of the corresponding Arrhenius regime.

\footnotetext{
${ }^{a)}$ E-mail: dirk.hegemann@empa.ch.

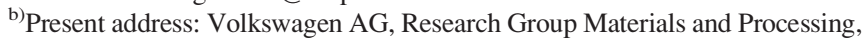
38436 Wolfsburg, Germany.

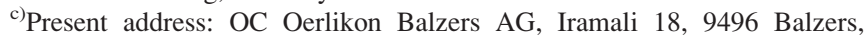
Liechtenstein.
}

Beside the flux of film-forming species, the film growth conditions are also affected by the energy flux incident on the substrate surface. The energy density $\varepsilon_{\text {surf }}$, i.e., the energy that dissipates within the growing film through ion bombardment, is then given by the energy flux per deposition rate $R$

$$
\varepsilon_{\text {surf }}=\frac{\Gamma_{i} E_{\text {mean }}}{R}
$$

with ion flux $\Gamma_{i}$ and mean ion energy $E_{\text {mean }}$. Note that Eq. (2) holds as long as deposition processes prevail over etching, since etched atoms also take up energy. Based on this approach, the plasma polymerization in capacitively coupled plasma (CCP) and inductively coupled plasma (ICP) discharges (on bias and floating potential) has recently been investigated. ${ }^{5,6}$

Similarly, the momentum density $\pi_{\text {surf }}$ during film growth can be defined as the "momentum flux" per deposition rate $R$

$$
\pi_{\text {surf }}=\sqrt{2 m_{i}} \frac{\Gamma_{i} \sqrt{E_{\text {mean }}}}{R}
$$

using

$$
E_{\text {mean }}=m_{i} v^{2}
$$

and the momentum

$$
m_{i} v=\sqrt{2 m_{i}} \sqrt{E_{\text {mean }}}
$$

where $m_{i}$ is the average ion mass and $v$ is the mean velocity of the energetic particles incident on the surface. The ion flux can be given by

$$
\Gamma_{i}=n_{0} v_{B} \cos \theta
$$

with the plasma density $n_{0}$, the Bohm velocity

$$
v_{B}=\sqrt{\frac{k T_{e}}{m_{i}}},
$$

and the collision factor 


$$
\cos \theta=0.86\left(3+\frac{d}{2 \lambda_{i}}\right)^{-0.5}
$$

where $d$ is the plasma length and $\lambda_{i}$ the ion mean free path. A good approximation of the mean energy of the incident particles considering collisions in the sheath is given by

$$
E_{\text {mean }}=e V_{s} \cos \varphi
$$

with the collision factor

$$
\cos \varphi=\left(1+\frac{c s}{\lambda_{i}}\right)^{-1}
$$

taking into account the width of the plasma sheath $s$ and the type of collisions ( $c=0.5$ for elastic collisions and somewhat higher for charge transfer). The momentum density is thus independent of the mass of the incident particles

$$
\pi_{\text {surf }}=\frac{n_{0} \sqrt{k T_{e} e V_{s}}}{R} \cos \Psi
$$

with

$$
\cos \Psi=\sqrt{2} \cos \theta \sqrt{\cos \phi} \approx \frac{\sqrt{2}}{2} \frac{1}{1+\Lambda p},
$$

where $\Lambda$ is a characteristic parameter of the plasma process comprising the expansion of the plasma zones (thus also depending on pressure $p$ ) and collision characteristics. Note that the pressure $p$ is inversely proportional to the mean free path $\lambda_{i}$, whereas plasma sheath $s$ and plasma length $l$ scale approximately with $p^{-1 / 3}$. For low energy input into the gas phase where the deposition rate increases almost linearly with power $W$ (energy-deficient regime), the momentum (and energy) density thus remains low (since the momentum flux scales roughly with $W^{5 / 4}$ ). With increasing energy input, on the other hand, the deposition rate gradually saturates (monomer-deficient regime). Both momentum density and energy density strongly increase, which might further be enhanced by a reduction of the deposition rate through ioninduced etching effects.

The "momentum flux" term in Eq. (6) is worth being further discussed. Considering that the electron density $n_{e}$ equals the plasma (ion) density $n_{0}$ for electropositive discharges, the momentum flux can be rewritten (in the collision-free case) as

$$
n_{0} \sqrt{k T_{e}} \sqrt{e V_{s}}=\sqrt{\left(n_{e} k T_{e}\right) \cdot\left(n_{0} e V_{s}\right)}
$$

which is the geometric mean of the energy density of electrons (integral moment of eedf) and the energy density of ions (integral moment of iedf). Hence, both the electron (eedf) and the ion energy distribution functions (iedf) determine the momentum flux in equal measure due to the initial condition for the acceleration of ions through the plasma sheath (Bohm criterion). Furthermore, the momentum density $\pi_{\text {surf }}$ represents the partial pressure exerted by the charged particles in the plasma on a surface which is modified by film growth. A typical partial pressure exerted on the surface by the charged particles (momentum flux) lies around $0.1 \mathrm{~Pa}$ in this work, which is $1 \%$ compared to the total pressure of $10 \mathrm{~Pa}$, although the ionization degree is only of the order of $10^{-5}$ (i.e., $\sim 10^{10} \mathrm{~cm}^{-3}$ electrons per $2.3 \cdot 10^{15} \mathrm{~cm}^{-3}$ particles). ${ }^{4}$ To break single molecular bonds, a threshold energy is thus required in the order of the bond dissociation energies (up to $6.3 \mathrm{eV}$ for $\mathrm{C}-\mathrm{C}$ ). The required energy is transferred by the energy density $\varepsilon_{\text {surf }}$, i.e., the energy flux acting on the surface of the growing film. 8,9

In order to investigate the densification of functional plasma polymers, pure ethylene $\left(\mathrm{C}_{2} \mathrm{H}_{4}\right)$ discharges as well as $\mathrm{CO}_{2} / \mathrm{C}_{2} \mathrm{H}_{4}(2: 1)$ and $\mathrm{NH}_{3} / \mathrm{C}_{2} \mathrm{H}_{4}(1: 1)$ mixtures were examined within a symmetric, capacitively coupled radio frequency (RF) plasma reactor (13.56 MHz excitation). ${ }^{10-12}$ The substrates were placed on the bottom electrode (diameter of $30 \mathrm{~cm}$ ) opposite to the gas inlet which was integrated in the plane-parallel top electrode with a gap of $5 \mathrm{~cm}$. The power input was varied from 5 to $150 \mathrm{~W}$, while gas flow rate $\left(8 \mathrm{sccm}\right.$ for $\left.\mathrm{C}_{2} \mathrm{H}_{4}\right)$ and pressure $(10 \mathrm{~Pa})$ were maintained. The excitation voltage and power absorption were measured by V/I probe (ENI Model 1065) and the electron density by MW interferometry (JE PlasmaConsult MWI 2650). The ion mean free path multiplied by pressure of $\mathrm{C}_{2} \mathrm{H}_{4}$ discharges was taken as $8.5 \mathrm{~mm} \cdot \mathrm{Pa}$ yielding collision factors (Eqs. (4)-(6)) as shown in Figure 1. XPS (PHI $5600 \mathrm{LS}$ ) was used to measure the chemical film composition and the density of $\mathrm{COOH}$ and $\mathrm{NH}_{2}$ functional groups after labeling with trifluoroethanol (TFE) and 4-trifluoromethyl-benzaldehyde (TFBA), respectively. ${ }^{13-15}$

The mass deposition rates of the three experimental series are depicted in Figure 2. The pure $\mathrm{C}_{2} \mathrm{H}_{4}$ discharge and the $\mathrm{NH}_{3} / \mathrm{C}_{2} \mathrm{H}_{4}$ (1:1) mixture show a broad Arrhenius regime (linear range in the Arrhenius-type plot) up to an energy density $\varepsilon_{\text {surf }}$ of about $25 \mathrm{eV}$ per deposited carbon atom, which can be derived from Eq. (2) by using the carbon atom density $\left(\right.$ per $\left.\mathrm{nm}^{3}\right) .^{3,5,6}$ At higher energy input, $W / F_{m}$, ion-induced etching effects reduce the deposition rate (according to increasing $\left.\varepsilon_{\text {surf }}\right)$. The $\mathrm{CO}_{2} / \mathrm{C}_{2} \mathrm{H}_{4}(2: 1)$ discharge reveals a slight reduction in deposition rate already at lower $W / F_{m}$ values independent of the energy density during film growth. ${ }^{10}$ This was ascribed to an increased formation of atomic oxygen by increasing fragmentation in the gas phase. ${ }^{4,10}$ In agreement, emission lines from atomic oxygen have been

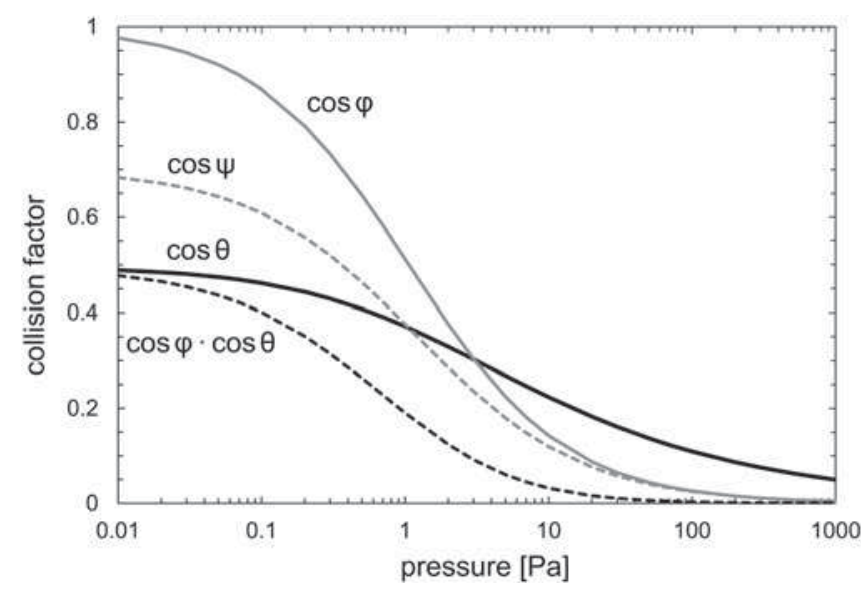

FIG. 1. Reduction of ion flux and ion energies incident on the electrode surface due to collisions upon increasing pressure. 


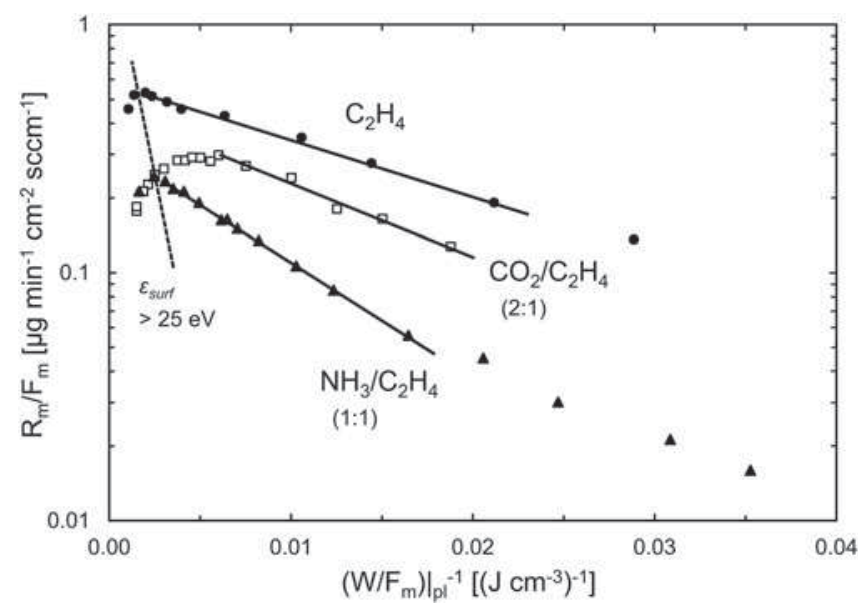

FIG. 2. Arrhenius-type plot of mass deposition rate per monomer gas flow, $R_{m} / F_{m}$, vs. the inverse energy input into the plasma, $\left.\left(W / F_{m}\right)\right|_{p l}{ }^{-1}$, for pure $\mathrm{C}_{2} \mathrm{H}_{4}$ discharges and admixtures of $\mathrm{CO}_{2}$ and $\mathrm{NH}_{3}$. The Arrhenius regime is indicated by straight lines. The dotted curve indicates where $\varepsilon_{\text {surf }}=25 \mathrm{eV}$ per deposited $\mathrm{C}$ atom is reached.

observed by optical emission spectroscopy (OES) for this regime, which are not present at lower energy input. Nevertheless, ion-induced effects might also contribute to the observed reduction in deposition rate with increasing energy density $\varepsilon_{\text {surf }}$. At low energy input a different plasma chemical reaction pathway might add to the deposition rate, thus showing a deviation from the Arrhenius regime as can be seen for the $\mathrm{NH}_{3} / \mathrm{C}_{2} \mathrm{H}_{4}$ (1:1) plasma.

Deposition rate beside electron density and sheath potential are then used to calculate the energy density $\varepsilon_{\text {surf }}$ and momentum density $\pi_{\text {surf }}$ during film growth for each experimental data point. The film density was calculated from the deposited mass and the film thickness. As shown in Figure 3, film density is found to be proportional to the momentum density for a certain range of energy densities. Within this range, film densification and cross-linking mainly take place via momentum transfer through energetic particle interaction.

Below the threshold energy density, which was found to be around $5-7 \mathrm{eV}$ per $\mathrm{C}$ atom, low values for the film density were obtained indicating low cross-linking and formation of

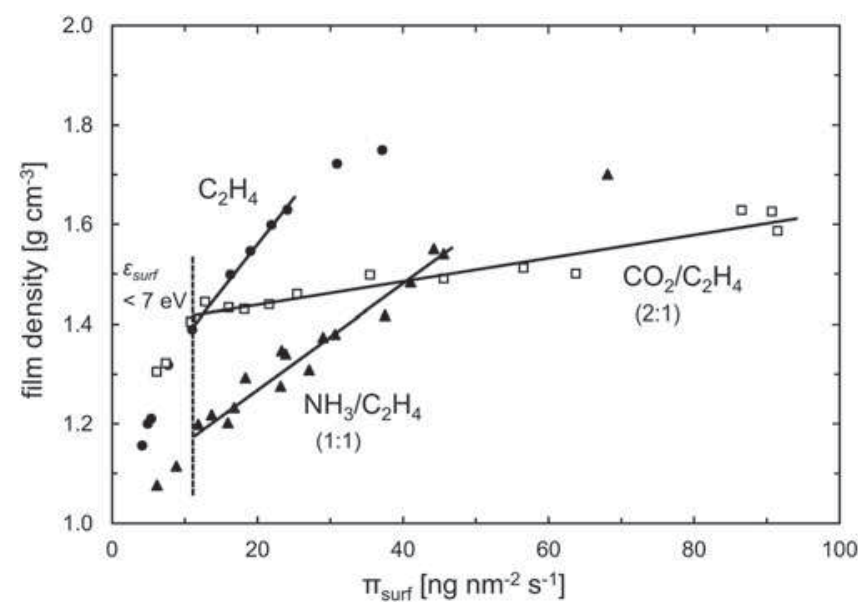

FIG. 3. Plasma polymer film density vs. the momentum density during film growth. The linear range indicates densification by momentum transfer.

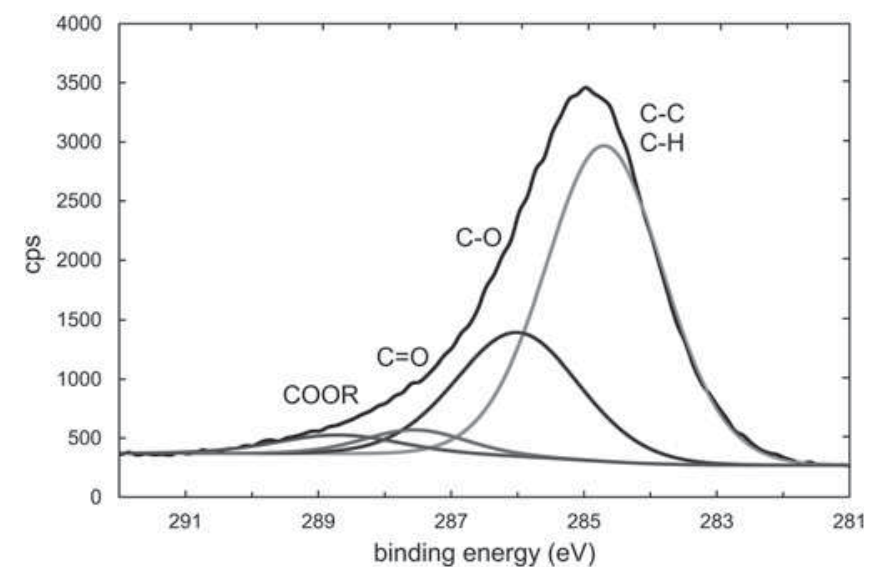

FIG. 4. XPS C1s spectrum of a a-C:H:O plasma polymer deposited with a $\mathrm{CO}_{2} / \mathrm{C}_{2} \mathrm{H}_{4}$ ratio of $2: 1$ using $30 \mathrm{~W}$ of power input.

numerous terminal groups $\left(-\mathrm{CH}_{3},-\mathrm{C}-\mathrm{OH},-\mathrm{C}=\mathrm{O},-\mathrm{C}-\mathrm{OOH}\right.$, $-\mathrm{C}-\mathrm{NH}_{2}$ ) yielding voids and rather unstable, swellable films. Above this threshold, permanent functional plasma polymers were obtained. The film density linearly scales with momentum density up to a certain energy density $(>25 \mathrm{eV}$ per deposited $\mathrm{C}$ atom) that enables ion-induced etching processes. The film density of a-C:H (using pure $\mathrm{C}_{2} \mathrm{H}_{4}$ discharges) increases by cross-linking and hydrogen abstraction finally yielding hard coatings, which also show relaxation effects (limiting the film density). ${ }^{3,16}$ Typically, $>50 \mathrm{eV}$ per deposited carbon atom was reported to yield the highest film density and hardness of a-C:H coatings. ${ }^{1,3}$ Likewise, the film densities of a-C:H:N increase, though at a lower rate. The low film densities around $1.2 \mathrm{~g} \mathrm{~cm}^{-3}$ indicate steric hindrance effects by incorporation of terminal $-\mathrm{NH}_{2}$ groups yielding nanoporous coatings. ${ }^{17}$ Abstraction of hydrogen and cross-linking via $\mathrm{C}$ and $\mathrm{N}$ atoms is thus required to achieve hard carbonitride coatings. The increase in film densities for a-C:H:O, on the other hand, was found to proceed much slower. Incorporation of $\mathrm{O}$ atoms thus limits the densification, since these cannot form 3-fold or 4-fold coordinated sites like $\mathrm{N}$ and $\mathrm{C}$, respectively.

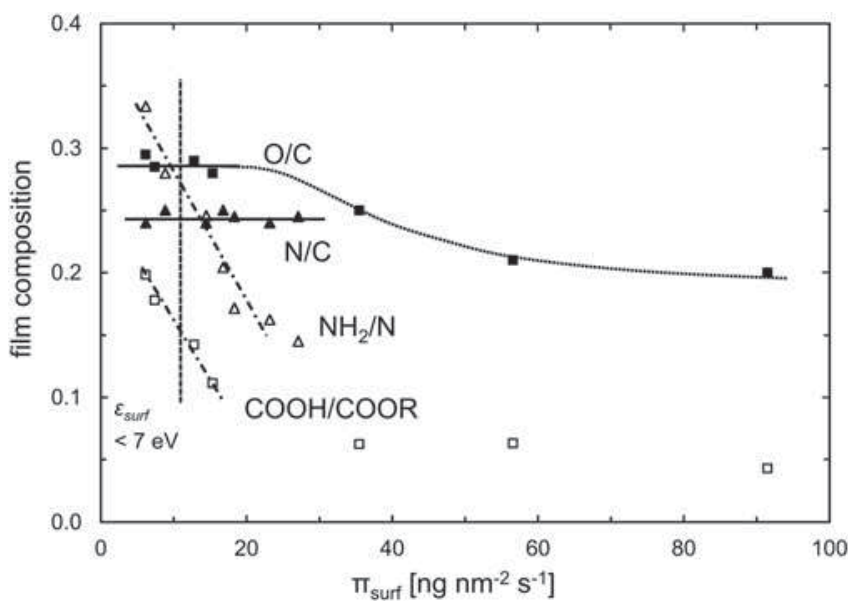

FIG. 5. Chemical composition of a-C:H:O and a-C:H:N films depending on the momentum transfer during film deposition. The filled symbols indicate the relative amount of oxygen and nitrogen, respectively, which is found to be constant within the Arrhenius regime. The open symbols show the functional group density which is reduced with increasing densification. 
The chemical composition of a-C:H:O and a-C:H:N was examined by XPS as shown in Figure 4. O/C and N/C ratios were found to be constant within the Arrhenius regime indicating a similar plasma chemical reaction pathway. Oxygen incorporation was only lowered at higher energies dissipated during film growth, where etching effects and momentum transfer are higher (Figure 5). Using derivatization methods the amount of carboxylic groups $(\mathrm{COOH})$ with respect to ester groups (COOR), thus showing esterification, can be defined as well as the amount of primary amino groups $\left(\mathrm{NH}_{2}\right)$ compared to the overall $\mathrm{N}$ incorporation. Both functional group densities inversely scale with densification as induced by momentum transfer.

We can thus conclude that the functionality of plasma polymer films strongly depends on the surface conditions such as energy and momentum density, while the C:O:N elemental composition can be maintained over a broad range of energy input into the gas phase (Arrhenius regime). Thresholds were observed both at low and high energies due to bond dissociation energies and ion-induced etching effects, respectively. These findings thus imply important principles for the optimization and up-scaling of functional plasma polymer depositions, which are of importance for diverse fields such as, e.g., textile-reinforced composites and tissue engineering.
${ }^{1}$ R. Kleber, M. Weiler, A. Krüger, S. Sattel, G. Kunz, K. Jung, and H. Ehrhardt, Diamond Relat. Mater. 2, 246 (1993).

${ }^{2}$ D. Music, U. Kreissig, Z. Czigany, U. Helmersson, and J. M. Schneider, Appl. Phys. A 76, 269 (2003).

${ }^{3}$ S. Peter, K. Graupner, D. Grambole, and F. Richter, J. Appl. Phys. 102, 053304 (2007).

${ }^{4}$ D. Hegemann, U. Schütz, and E. Körner, Plasma Process. Polym. 8, 689 (2011).

${ }^{5}$ D. Hegemann, E. Körner, S. Chen, J. Benedikt, and A. von Keudell, Appl. Phys. Lett. 100, 051601 (2012).

${ }^{6}$ J. Trieschmann and D. Hegemann, J. Phys. D: Appl. Phys. 44, 475201 (2011).

${ }^{7}$ J. Meichsner, K. Dittmann, and C. Küllig, Contrib. Plasma Phys. 52, 561 (2012).

${ }^{8}$ P. Favia, R. Lamendola, and R. d'Agostino, Plasma Sources Sci. Technol. 1, 59 (1992).

${ }^{9}$ A. von Keudell, W. Jacob, and W. Fukarek, Appl. Phys. Lett. 66, 1322 (1995).

${ }^{10}$ D. Hegemann, E. Körner, K. Albrecht, U. Schütz, and S. Guimond, Plasma Process. Polym. 7, 889 (2010).

${ }^{11}$ E. Körner, G. Fortunato, and D. Hegemann, Plasma Process. Polym. 6, 119 (2009).

${ }^{12}$ S. Guimond, U. Schütz, B. Hanselmann, E. Körner, and D. Hegemann, Surf. Coat. Technol. 205, S447 (2011).

${ }^{13}$ P. Favia, M. V. Stendardo, and R. d'Agostino, Plasma Polym. 1, 91 (1996).

${ }^{14}$ P. L. Girard-Lauriault, F. Mwale, M. Iordanova, C. Demers, P. Desjardins, and M. R. Wertheimer, Plasma Process. Polym. 2, 263 (2005).

${ }^{15}$ E. Körner, P. Rupper, J. F. Lübben, A. Ritter, J. Rühe, and D. Hegemann, Surf. Coat. Technol. 205, 2978 (2011).

${ }^{16}$ C. A. Davis, Thin Solid Films 226, 30 (1993).

${ }^{17}$ S. Guimond, B. Hanselmann, M. Amberg, and D. Hegemann, Pure Appl. Chem. 82, 1239 (2010). 